\title{
Stationäre Versorgung von Diabetikern soll besser werden
}

\author{
Etwa 25\% bis 30\% der Krankenhauspatienten haben ei- \\ nen schlecht eingestellten Blutzucker und somit ein ho- \\ hes Komplikationsrisiko. Zur besseren Versorgung dieser \\ Patienten hat Lilly Diabetes die „Diabetes-Excellence- \\ Center"(DEC)-Initiative ins Leben gerufen.
}

Mit der „Diabetes-Excellence-Center“ (DEC)-Initiative soll unter anderem ein diabetesspezifisches Struktur- und Prozessmanagement in Kliniken etabliert werden.

„Für Patienten mit Diabetes ist die Versorgung in deutschen Krankenhäusern in den letzten Jahren eher schlechter als besser geworden“, erklärte PD Dr. Erhard Siegel, Chefarzt der Abteilung für Gastroenterologie, Diabetologie und Ernährungsmedizin im St. Josefskrankenhaus in Heidelberg. Er sieht eine Ursache in den fehlenden Absprachen, wenn mehrere Fachdisziplinen involviert sind. Insbesondere Patienten mit der Nebendiagnose Diabetes mellitus werden bei Einlieferung anlässlich chirurgischer oder kardiologischer Diagnosen oft nicht adäquat versorgt. Die Folgen sind Wundheilungsstörungen und systemische Infektionen sowie längere Verweilzeiten im Kranken- haus. An negativen Auswirkungen hinzu kommen - aus Sicht des Krankenhausmanagements - entgangene Erlöse für eine spezielle antidiabetische Versorgung.

\section{Module für eine verbesserte Behandlungsqualität}

In Zusammenarbeit mit dem Berufsverband der Diabetologen in Kliniken e.V. (BvDK) und der Deutschen Diabetes-Gesellschaft (DDG) hat Lilly „DEC“ initiiert und verschiedene Module für eine bessere Behandlungsqualität erarbeitet. $\mathrm{Zu}$ den Bausteinen gehören Workshops und praxisnahe Materialien wie Implementierungsleitfäden, SOP („Standard Operating Procedure“)-Muster oder Konsilbögen für die stringente Diagnostik und Dokumentation in allen Klinikabteilungen. Zudem wurde ein spezieller Kodierleitfaden für Diabetes entwickelt. Er hilft
Schritt für Schritt bei der richtigen Verschlüsselung. Eine weitere Hilfestellung für eine leitliniengerechte Diabetestherapie gibt das „Diabetes mellitus XXS pocket“, ein kompaktes Nachschlagewerk im Kitteltaschenformat.

Mittlerweile haben Vertreter von mehr als 200 Kliniken an den eintägigen Workshops teilgenommen. Die Resonanz sei beachtlich, betonte Siegel, zumal die Zahl diabetologischer Lehrstühle und Ausbildungsstätten in Deutschland rückläufig sei. Auch viele Geschäftsführungen erkennen in den Entwicklungen einen hohen Wert - zum Vorteil der Patienten.

Dr. Ellen Jahn, Bad Homburg

Workshop „Verbesserung der klinischen Versorgung von Diabetespatienten für Geschäftsführung, Pflege, Ärzte"; 11.10.2012, Bad Homburg; Veranstalter: Lilly Deutschland GmbH.

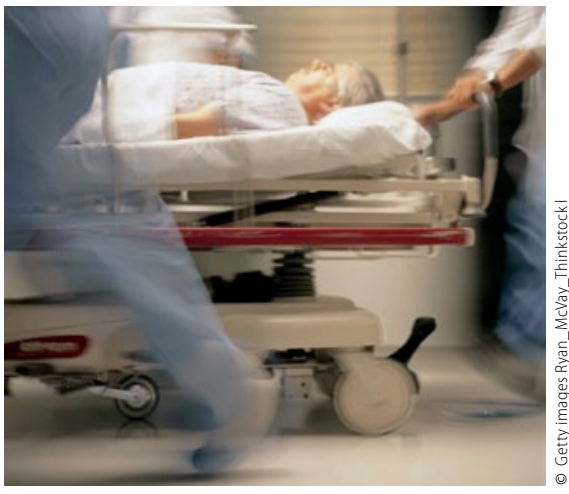

Nicht immer wird die Nebendiagnose Diabetes bei einer stationären Therapie hinreichend berücksichtigt.

\section{Kardiometabolische Vorteile}

\author{
Die Hemmung des renalen Glukosetransporters SGLT-2 \\ ist ein neuer antidiabetischer Ansatz. Die Wirkstoffe sind \\ unkompliziert einzunehmen und haben einen günsti- \\ gen Einfluss auf Körpergewicht und Blutdruck.
}

„SGLT-2-Hemmer senken insulinunabhängig die Nierenschwelle für Glukose“, sagte Prof. Jochen Seufert vom Uniklinikum Freiburg. Dadurch scheiden die Patienten deutlich mehr Glukose aus, was $\mathrm{zu}$ einem $\mathrm{HbA}_{1 \mathrm{c}}$-Abfall und $\mathrm{zu}$ einer durch den Kalorienverlust bedingten Körpergewichtsabnahme führt. Systoli- scher und diastolischer Blutdruck sinken ebenfalls. Das sei v.a. ein Natrium-Effekt, so Seufert. Da SGLT-2 zusammen mit Glukose auch Natrium aus den Nierentubuli rückresorbiert, führt seine Hemmung zu vermehrter Natriumausscheidung, ähnlich einem milden Diuretikum. Seufert präsentierte Daten aus klinischen
Studien zu Canagliflozin, die zeigen, dass dieser Wirkstoff in der Monotherapie und als Add-on zu oralen Antidiabetika oder Insulin $\mathrm{HbA}_{1 \mathrm{c}}$-Wert, Gewicht und Blutdruck senkt. Günstig bewertete Seufert zudem, dass das Hypoglykämierisiko bei SGLT-2-Hemmung sehr gering ist. Auch das Sicherheitsprofil der Gliflozine sei insgesamt günstig. Beschrieben ist vermehrtes Auftreten von Genitalinfektionen. Harnwegsinfekte träten den bisherigen Daten zufolge nicht vermehrt auf.

Prof. Stephan Jacob, Villingen-Schwenningen, wies auf experimentelle Daten hin, wonach Gliflozine die Betazellfunktion bessern könnten, was noch zu verifizieren ist.

Philipp Grätzel, Berlin

Quelle: Satellitensymposium DDG-Herbsttagung, 16.11.2012, Berlin, Veranstalter: Janssen-Cilag 